\title{
In-vitro-Diagnostik im Fokus
}

$M$ ehrere Beiträge zur In-vitroDiagnostik in der Allergologie finden sich in dieser Ausgabe, die damit pünktlich vor dem 1 . April dieses Jahres auf eine wichtige Änderung im allergologischen Labor hinweist: Ab diesem Datum gilt nämlich die neue - bereits am 15. Februar 2008 im Deutschen Ärzteblatt veröffentlichte „Richtlinie der Bundesärztekammer zur Qualitätssicherung laboratoriumsmedizinischer Leistungen“. Die wichtigsten Veränderungen sind die obligate Einführung eines Qualitätsmanagementsystems sowie interner und unter bestimmten Voraussetzungen auch externer Qualitätskontrollen. Die Details hat Prof. Dr. Harald Renz auf Seite 94 in diesem Heft für Sie zusammengefasst.

Offen bleibt dabei vorerst noch die Frage, ob die Bestimmung des spezifischen IgE als qualitative oder als quantitative Untersuchung anzusehen ist, da die bisherige Einstufung als semiquantitativ nicht mehr vorgesehen ist. Prinzipiell würde die Angabe des Ergebnisses in CAP-Klassen oder ähn-

„Die Entscheidung darüber, ob die Bestimmung des spezifischen IgE jetzt als qualitative oder als quantitative Untersuchung anzusehen ist, sollte nicht nur vom technisch Machbaren, sondern auch vom allergologisch Notwendigen bestimmt sein.“ lichen Bewertungen eine qualitative Bestimmung darstellen, während die Angabe in kU/l eine quantitative Bestimmung wäre. Hier bestünde auch die Option, dass der für die Bestimmung Verantwortliche die Entscheidung entsprechend den diagnostischen Bedürfnissen trifft.

In kommenden Diskussionen bei der Bundesärztekammer wird sich zeigen, ob für diese Untersuchungen neben einer internen Kontrolle auch eine externe Kontrolle durch Ringversuche notwendig wird.

Gleichzeitig finden Sie in dieser Ausgabe die Neuedition der Leitlinie der allergologischen Fachgesellschaften zur In-vitro-Diagnostik, die neue Entwicklungen seit dem Erscheinen der ersten Leitlinie reflektiert. Federführender Autor ist hier ebenfalls Herr Renz (S. 110).

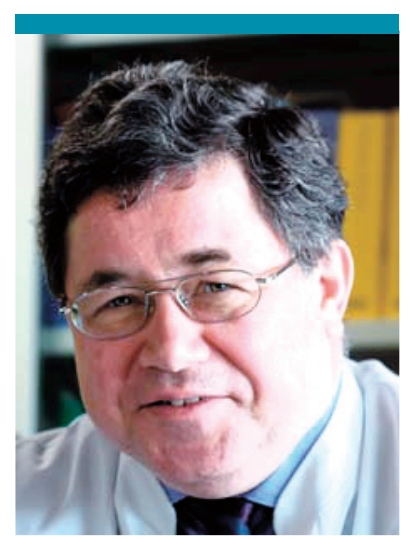

Prof. Dr.Hans F. Merk, Hautklinik der Medizinischen Fakultät, Universitätsklinikum der RWTH Aachen

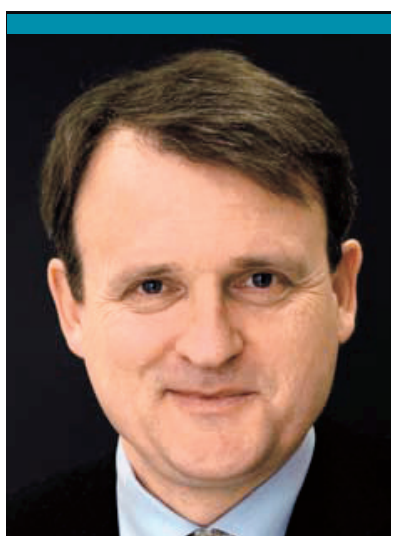

Prof. Dr. Thilo Jakob, Allergieabteilung \& Forschergruppe Allergologie, UniversitätsHautklinik Freiburg
Es bleibt zu hoffen, dass die Versuche zur Qualitätssicherung der Laboruntersuchungen vor dem Hintergrund der gegenwärtigen Erstattung allergologischer Leistungen nicht zur weiteren Reduktion allergologischer Diagnostik führt. Dabei sollte die Entscheidung darüber, ob die Bestimmung des spezifischen IgE jetzt als qualitative oder als quantitative Untersuchung anzusehen ist, nicht nur vom technisch Machbaren, sondern auch vom allergologisch Notwendigen bestimmt sein. In den meisten Fällen wird in der Diagnostik der qualitativ angegebene Wert ausreichen. Es stellt sich doch vielmehr die Frage, ob nicht die Ausweitung der qualitativen Diagnostik durch Berücksichtigung neuer Methoden, wie beispielsweise dem Einsatz rekombinant gewonnener Allergene oder der Verwendung von Microarray-Analysen, mehr klinisch fassbaren Fortschritt bedeuten würde als die Möglichkeit das spezifische IgE quantitativ exakt angeben zu können. Lektüre.

Wir wünschen Ihnen eine anregende

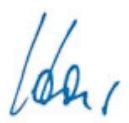<smiles>CCCCC=C(C)C</smiles>

Prof. Dr. Hans F. Merk
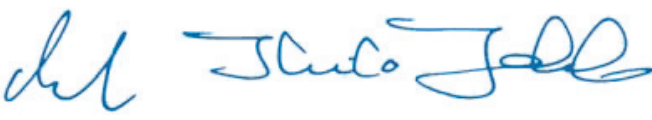

Prof. Dr. Thilo Jakob 